Side Area-Assisted 3D Evaporator with Antibiofouling Function for Ultra-Efficient Solar

\title{
Steam Generation
}

Haoxuan Li, ${ }^{1,2,3,4} \dagger$ Wei Zhu, ${ }^{1,2,4} \dagger$ Meng Li, ${ }^{1,2,4}$ Ying Li, ${ }^{1 *}$ Ryan T. K. Kwok, ${ }^{4}$ Jacky W. Y. Lam, ${ }^{4}$ Lei Wang, ${ }^{1,2}$ Dong Wang, ${ }^{1 *}$ Ben Zhong Tang ${ }^{4 *}$

\section{Affiliations}

1 Centre for AIE Research, Shenzhen Key Laboratory of Polymer Science and Technology, Guangdong Research Center for Interfacial Engineering of Functional Materials, College of Material Science and Engineering, Shenzhen University, Shenzhen 518061, P. R. China

2 Key Laboratory of Optoelectronic Devices and Systems of Ministry of Education and Guangdong Province, College of Physics and Optoelectronic Engineering, Shenzhen University, Shenzhen 518061, P. R. China

3 Key Laboratory of Eco-Textiles (Ministry of Education), Nonwoven Technology Laboratory, Jiangnan University, Wuxi 214122, China

4 Department of Chemistry, The Hong Kong University of Science and Technology, Clear Water Bay, Kowloon, Hong Kong, China

$\dagger$ These authors contributed equally to this work

*Corresponding author. Email: wangd@szu.edu.cn, liyingway@ szu.edu.cn, tangbenz@ust.hk

\section{Teaser}

We report a porous all-fiber porous cylinder like foam (AFPCF) containing AIE luminogen (AIEgens) with superior capacities of photothermal conversion and ROS generation to simultaneously realize efficently solar steam generation and antibiofouling effects.

\section{Abstract}

Solar-driven interfacial steam generation (SISG) has been recognized as the most promising strategy to solve water shortages in an eco-friendly and low-cost way. However, the practical application of SISG is vitally restricted by some inherent limits, especially for finite evaporation rate and insufficient working life of evaporator. Herein, we explore a novel SISG system involving an all-fiber porous cylinder-like foam (AFPCF) 3D evaporator, side area-assisted evaporation protocol, and aggregation-induced emission (AIE)-active molecules with "one stone two birds" function. The AIE-featured solar absorber exhibits highly efficient sunlight absorption and photothermal conversion, endowing the side area-assisted evaporator with as high as $3.6 \mathrm{~kg}$ 
$37 \mathrm{~m}^{-2} \mathrm{~h}^{-1}$ of solar evaporation rate under 1 sun of irradiation. Moerover, the evaporator is capable of 38 powerfully producing reactive oxygen species (ROS) upon sunlight irradiation benefiting the prominent photosensitizing property of the AIE molecules, which results in extraordinary photodynamic killing of bacteria nearby the fiber to prevent biofouling, consequently improving the working life of evaporator.

\section{Introduction}

Solar driven interfacial steam generation (SISG) is a desirable strategy to continuously produce clean water by utilizing sea water and solar energy, which both are considered as inexhaustible recourses on the earth. (1-3) Of particular importance, SISG is indeed a zero-carbon emission process, making it a great potential candidate to alleviate the global water scarcity. $(4,5)$ Over the past decades, enthusiastic efforts have been devoted by scientists on designing ideal evaporator, comprised of solar absorber for efficient light-to-heat conversion as well as floating structure for thermal insulation and water transportation, to increase the evaporation rate. (6-8) Compared to two-dimensional (2D) evaporator, three-dimensional (3D) analogues exhibit excellent evaporation performance due to the reduced light reflection and gaining energy from the environment. $(9,10)$ For example, by increasing the height of the cylindrical cup-shaped structrure, the evaporator can generate vapor with an evaporation rate of $2.04 \mathrm{~kg} \mathrm{~m}^{-2} \mathrm{~h}^{-1}$ under the irraidation of $1 \mathrm{sun}$, far exceeding that for 2D structure $\left(1.21 \mathrm{~kg} \mathrm{~m}^{2} \mathrm{~h}^{-1}\right)$. (11) Another typical 3D cone structure can achieve an evaporation rate of $1.7 \mathrm{~kg} \mathrm{~m}^{-2} \mathrm{~h}^{-1}, 1.7$ times higher than that of planar film. (12) However, nearly all of the previous works set the irradiaiton of simulated sun in vertical direction during evaporation test, while the irradiation in tilt direction had been rarely investegated. Inspired by that, side area-assisted evaporation attracted our attention for designing 3D evaporator, wherein both surface area and side area of the evaporator can absorb solar energy under natural sunlight due to its tilt irradiation function, which could consequentially amplify both effective heating area and evaporation rate. Hence, side area-assisted evaporation may offer a feasible and simple protocol to design advanced evaporator to beyond the limition of evaporation efficiency.

The solar absorber can be categorized as plasmonic materials, carbon-based materials and organic molecules. (13-18) Among them, plasmonic and carbon-based absorbers have their inherent weaknesses, such as low chemical stability and high cost for plasmonic absorbers, and low stability against water and bacteria for carbon absorbers. (19-21) Besides, those developed 
71 systems generally feature a single function with transfering solar energy into heat, and 72 multifunctional evaporator remain unexploited but supremely desirable. Especially, during evaporation, the formed warm environment around the evaporator is capable of promoting the growth of microorganisms which seriously affects working life of the evaporator. Therefore,

exploiting functional absorber to simultaneously achieve efficient evaporation and antibiofouling property is a major requirement. (22, 23) In conventional methods, $\mathrm{Ag}$, (20) $\mathrm{ZnO}$ (21) nanoparticles and Mxene (24) are additionally dopped into evaporator as bacteriostatic agent, endowing the evaporator with antibioflouling behavior to provent channel plugging caused by microorganisms proliferation. Those strategies, however, require tedious labor in the preparation process and influence evaporation output due to the insufficient compatibility. Evidently, the exploration of a single material sharing both photothermal conversion and antibiofouling capacites would be an appealing yet significantly challenging task. In the circumstances, as a newly emerged photosensitizing agent, aggregation-induced emission (AIE)-active molecule that could simultaneously afford high performance photothermal conversion and eactive oxygen species (ROS) prodcution through rational design, (25-28) could be an ideal candidate for constrcting 3D evaporator with efficient evaporation and antibiofouling functions.

In this work, we report a porous all-fiber porous cylinder like foam (AFPCF) containing AIE luminogen (AIEgens) with superior capacities of photothermal conversion and ROS generation to simultaneously realize efficently solar steam generation and antibiofouling effects (Fig.1A). Side area-assisted evaporation and antibiofouling activity is for the first time integrated into an evaporator. This presented evaporator features an interconnected porous structure with excellent hydrophilicity for vapor escaping and water suppling, side-area assisted evaporation system for increased effictive evaporation area, as well as efficient photodynamic killing of bacteria nearby the fiber to prevent biofouling, collectively offering a design blueprint for the next generation of solar steam production materials.

\section{Results and Discussion}

\section{Fabrication and characterization of AFPCF}

As shown in Fig. 1A, a typical D-A-D molecule (TPA-BTDH) were well designed and facilely synthesized in three steps (Fig. S1). In the primary step, Suzuki-Miyaura coupling reaction smoothly proceeded by employing 4-methoxy-N-(4-methoxyphenyl)-N-(4-(4,4,5,5-tetramethyl1,3,2-dioxaborolan-2-yl)phenyl)aniline and 4,7-dibromo-5,6-dinitrobenzo[c][1,2,5]thiadiazole as starting materials, producing TPA-BTDNO with a yield of 53.2\%. Iron-catalyzed nitro reduction of TPA-BTDNO was next conducted in the presence of acetic acid and iron powder, yielding intermediate product TPA-BTDNH. Subsequent cyclization with benzils to obtain the TPA- 
BTDH with a yield of $95.6 \%$. The intermediates and final compounds are characterized by ${ }^{1} \mathrm{H}$ NMR, ${ }^{13} \mathrm{C}$ NMR, and high-resolution mass spectrum (HRMS). The single crystal of TPA-BTDH was obtained in chloroform/hexane under slow evaporation. As shown in Fig. 1B and Table. S1, the intermolecular distances between two parallel planes were as large as $3.609 \AA$ and $6.702 \AA$ respectively, which exceeding the typical $\pi-\pi$ stacking that usually quenches the fluorescence. The twisted angles between the acceptor TQ and donor MTPA of TPA-BTDH were $51.8^{\circ}$, and the other dihedral angles inside the molecule were $69.71^{\circ}$ and $72.4^{\circ}$, and $58.81^{\circ}$, respectively (Fig. $\mathrm{S} 2 \mathrm{~A})$. All these features of TPA-BTDH including abundant molecular rotators, large twisted angles and the nonplanar structure, may enable its intramolecular motions to be partially restricted but still active in fiber state, potentially allowing high photothermal conversion and ROS generation benefiting from the balanced energy dissipations (Fig. 1C).

As illustrated in Fig. 1D, the maximum absorption wavelength of TPA-BTDH was located at $645 \mathrm{~nm}$ and the photoluminescence (PL) spectra of TPA-BTDH were peaked at $908 \mathrm{~nm}$ in the NIR-II region. The strong and broad absorption is beneficial to absorbing the light energy of sunlight. In addition, the long-wavelength absorption was detremined to be in good accordance with the narrow HOMO-LUMO bandgap of (1.702 eV) (Fig. S2B). Subsequently, the photosensiting properties of TPA-BTDH were investigated in terms of ROS production and photothermal conversion. It was observed that the emission intensity of ROS indicator DCFH-DA was remarkably boosted with a 195-fold enhancement, revealing the high efficiency of ROS generation (Fig. 1E). Upon solar irradiation of 1 sun, the temperature of TPA-BTDH powder increased with time and reached a maximum of $62.8{ }^{\circ} \mathrm{C}$ within $150 \mathrm{~s}$, suggesting its excellent capacity of transferring solar energy to heat (Fig. 1F).

Encouraged by the excellent properties of TPA-BTDH, 3D-architecture nanofibrous mat with TPA-BTDH as solar absorber was then fabricated by using gas-forming technology. $(17,29)$ Firstly, TPA-BTDH were dopped into fiber during electrospinning. The absorber would be stable given that TPA-BTDH was wrapped in the polymethyl methacrylate (PMMA) rather than coating on the matrix surface (Fig. S3A and S3B). Even though their absorption was limited in the range from 550 to $1000 \mathrm{~nm}$ (Fig. S3C), the TPA-BTDH/PMMA nanofibrous mat had over 80\% of light absorption and showed considerable capacity for transferring solar to heat. The temperature of the mat could increase to $61.5^{\circ} \mathrm{C}$ under $150 \mathrm{~s}$ of 1 sun irradiation (Fig. S3D). Subsequently, the TPABTDH/PMMA nanofibrous mats were cut into a circle with the diameter of $3 \mathrm{~cm}$, treated by plasma for improving their hydrophilic, and then expanded in the $\mathrm{NaHB}_{4}$ solution (1M) for different time (Fig. 2A). With the gas bubble generated and escaped through the mats (Fig. 2B), thickness changes of the nanofibrous mat following expansion were shown in Fig. 2C. Evidently, 
139 the height of expanded 3D AFPCF increased from 1 to 3 and $5 \mathrm{~cm}$, as the expansion time increased from 10 to 30 and $60 \mathrm{~min}$, respectively. To be noted that, the hydrophilicity of the 2D nanofibrous mats given by plasma treatment is the key factor for expanding into 3D structure. Aiming to investigate the relationship between side area and evaporation rate, we prepared three groups 3D AFPCF with the height of 1,3 and $5 \mathrm{~cm}$, and the corresponding effective areas were about 10, 16 and $22 \mathrm{~cm}^{2}$, respectively (Fig. 2D). The scanning electron microscope (SEM) images revealed that gap between each layer of nanofiber was observed to increase with raising expansion time, and the thickness of layer was decreased (Fig. 2E and Fig. S4). Such all-fiber structure bearing both interconnected pore and superior hydrophilicity endowed the 3D AFPCF with water transportation and vapor escape functions. As depicted by UV-vis-NIR absorption spectrum of the 3D AFPCF in Fig. 2F, the 3D AFPCF exhibited efficient absorption in the range from 500 to $1000 \mathrm{~nm}$, which is the main light energy band of natural sun, suggesting that the 3D AFPCF possesses excellent capacity to absorb sunlight. It was obserevd that the temperature of AFPCF in dry state rose to $59.2{ }^{\circ} \mathrm{C}$ within 10 min upon 1 sun irradiation (Fig. S5). The high localized temperature on the surface of the AFPCF is beneficial for solar steam generation. (30)

\section{Side area assisted evaporation}

To explore its solar steam generation ability, AFPCF with the height of $3 \mathrm{~cm}$ was wrapped by foam and then floated on a beaker filled with $3.5 \mathrm{wt} \% \mathrm{NaCl}$ solution in water (Fig. 3A). The temperatures of the evaporator surface and side areas were also recorded by IR camera to investigate the effect of the irradiation direction of the simulated sunlight on the evaporation capacity (Fig. 3B and 3C). As the irradiation direction was set as vertical to the evaporator, the temperature of evaporator surface was increased to $46.5{ }^{\circ} \mathrm{C}$ within $1 \mathrm{~h}$, which was much higher than that of the evaporator side (only $28.9^{\circ} \mathrm{C}$ ). In addition, as the irradiation direction was tilted to the evaporator (with incident angle of $60^{\circ}$ ), the temperatures of evaporator surface and side were increased to $44.5^{\circ} \mathrm{C}$ and $39.5^{\circ} \mathrm{C}$ within $1 \mathrm{~h}$, respectively. Therefore, both surface and side of the evaporator acted as heated evaporation area to generate vapor during solar tilt irradiation. In this way, the effective evaporation area can be greatly increased without the increase of the evaporator surface. The mass of simulated seawater decreased with the increased of irradiation time (Fig. 3D). Under the vertical irradiation of 1 sun, the evaporation rate of the evaporator was determined to be $2.4 \mathrm{~kg} \mathrm{~m}^{-2} \mathrm{~h}^{-1}$, and the corresponding dark evaporation rate was $0.8 \mathrm{~kg} \mathrm{~m}^{-2} \mathrm{~h}^{-1}$. Notably, as the vertical irradiation was turned to the tilted irradiation, a dramatic increase in the evaporation rate from 2.4 to $3.6 \mathrm{~kg} \mathrm{~m}^{-2} \mathrm{~h}^{-1}$ was revealed mainly due to the additional heat generated by the side area, and the high evaporation rate remained stable over time (Fig. 3E). 
172 These results indicated that side area-assisted evaporation is a facile strategy to enhance the overall evaporation based on the natural sunlight tilting irradiation.

To systematically investigate the effect of side area on the evaporation performance, the evaporators with height of 1 and $5 \mathrm{~cm}$ were also tested under the vertical and tilted irradiation of 1 sun (Fig. S6). Under the vertical and tilted irradiation for $1 \mathrm{~h}$, the surface temperature of the 1 cm-height evaporator reached at 42.2 and $41.5{ }^{\circ} \mathrm{C}$, respectively (Fig. S7), while its side temperature was not recorded, since the side area was not sufficient enough to absorb solar energy. In the case of $5 \mathrm{~cm}$-height evaporator, upon vertical and tilted irradiation for $1 \mathrm{~h}$, the surface temperatures were measured to be 43.3 and $42.5{ }^{\circ} \mathrm{C}$, respectively, while the side temperatures were determined to be 28.2 and $36.2{ }^{\circ} \mathrm{C}$ (Fig. S6C). Moveover, the corresponding evaporation rates were calculated to be 1.6 and $1.8 \mathrm{~kg} \mathrm{~m}^{-2} \mathrm{~h}^{-1}$ for the $1 \mathrm{~cm}$-height evaporator, and 2.8 and 3.2 $\mathrm{kg} \mathrm{m}^{-2} \mathrm{~h}^{-1}$ for the $1 \mathrm{~cm}$-height evaporator, under the vertical and tilted irradiation of 1 sun (Fig. $\mathrm{S} 6 \mathrm{D}$ and $6 \mathrm{E})$, respectively. These results indicated that the evaporation rate can be significantly enhanced by increasing the height of the evaporator. It was also found that the evaporation rate of $5 \mathrm{~cm}$-height evaporator was lower than that of the $3 \mathrm{~cm}$-height evaporator, which can be attributed to the restrained water transportation in the case of $5 \mathrm{~cm}$-height evaporator. This issue could be solved by constructing 3D smart floating structure, which can transport water to higher altitude. According to classical energy efficiency calculations, all the evaporators with different side areas showed the evaporation rate beyond the theoretical limit of about $1.47 \mathrm{~kg} \mathrm{~m}^{-2} \mathrm{~h}^{-1}$, which can be attributed to the additional energy harvest by the side area which absorbed the sunlight. Indeed, the presented evaporator remarkably offers a design philosophy to significantly enhance the evaporation performance.

\section{Antibiofouling performance}

Working life is a key criterion to estimate a evaporator. As known that a warm environment around the evaporator can be formed during the evaporation process, and promote the growth of microorganisms, which seriously affects the working life of evaporator. Inspired by the efficect ROS generation of TPA-BTDH, as well as high effciency of bacteria photodynamic killing, the antibiofouling effect of the AFPCF was assessed by attaching four typical bacteria, Escherichia coli (E. coli), Staphylococcus epidermidis (S. epidermidis), Staphylococcus aureus (S. aureus) and methicillin-resistant Staphylococcus aureus (MRSA) on the evaporator surfac, respectively. For the contact-kill test, the blank control and AFPCF samples with a diameter of $1.5 \mathrm{~cm}$ were

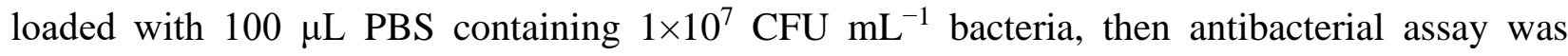
assessed by agar plate counting. For accuracy, six parallel samples were placed on each group. As shown in Fig. 3F, four types of bacteria grow well in the blank control plate after the radiation of 
the simulated sunlight for $10 \mathrm{~min}$. In contrast, within $10 \mathrm{~min}$ of irradiation, $99.86 \% \mathrm{E}$. coli, 99.91\% S. epidermidis, $99.96 \%$ S. aureus and 99.98\% MRSA were killed rapidly by AFPCF, owing to its superior capability to generate ROS under visible light (Fig. S8). Moreover, considering that the AFPCF is easy to generate ROS incessantly under the sunlight, enabling the evaporator to suppress attachment and proliferation of bacteria and/or microorganisms on the nanofiber, resulting in good antibiofouling of the evaporator. We also used E. coli as a model to investigate the antibacterial performance of the AFPCF at alternate day and night. Each cycle includes three stages, pipetting $1 \times 10^{6} \mathrm{CFU} \mathrm{mL}{ }^{-1}$ E.coli on the AFPCF, incubating them in dark condition for $50 \mathrm{~min}$ and exposing them to simulated sunlight for $10 \mathrm{~min}$. Five cycles of the antibacterial result indicated that the biocidal efficacy of the AFPCF maintained constance with a 99.9\% inhibiting rate against E. coli (Fig. 3G). Moreover, it was revealed that the AFPCF cannot generate ROS at night, due to the lack of light to drive molecule excitation, revealing the weakly antibacterial capacity in dark condition (Fig. S9). This cycle test also suggested that the bacteria accumulated on the nanofiber at night can be killed at daytime, showing excellent service life with self-antibiofouling property.

The morphological changes of bacteria on the nanofiber with or without light irradiation were visualized by SEM. Both E. coli and S. aureus exhibited rod and spherical shape with smooth surface in the darkness. As shown in Fig. S10, the cellular destruction and surface wrinkled of the E. coli together with the lesions of $S$. aureus were observed on the surface of the nanofiber after the AFPCF exposure to $10 \mathrm{~min}$ of simulated sunlight irradiation. This observation indicated that the bacteria on the nanofiber can be easily killed via disrupting bacterial cell membranes, which is similar to peroxide disinfectants. Apart from killing the bacteria attached on the nanofiber, the AFPCF can also kill the bacteria nearby the evaporator, offering a facile strategy to clean up the polluted lake and/or river. In additon, the antibacterial activity of the AFPCF under natural sunlight irradiation was also investigated. As illustrated in Fig. S11, each well of the six-well plate was pipetted $5 \mathrm{~mL}$ PBS solution containing $10^{7} \mathrm{CFU} \mathrm{mL} \mathrm{m}^{-1}$ bacteria, and three groups of $E$. coli, $S$ aureus and MRSA were tested, respectively. The AFPCF was placed on three wells, and other three wells was set as blank control. Under the irradiation of natural sunlight with $0.76 \mathrm{~kW}$ $\mathrm{m}^{-2}$ for $1 \mathrm{~h}(2: 00 \mathrm{PM}$ to 3:00 PM), viable colonies of E. coli, $S$ aureus and MRSA grew well on the plates in the absence of AFPCF, whereas a significant decrease in all types of bacteria survival occurred in the presence of AFPCF, which should be attributed to the ROS generation. Owing to excellent insulation performance of the AFPCF, there is no significant changes in temperature of PBS solution with or without AFPCF under the irradiation of sunlight. Hence, ROS played a key role in killing bacteria. Limited by the operating distance of ROS, only the bacteria near the 
evaporator can be efficiently killed, which is beneficial to treat surface water pollution without affecting on fish or other organisms living in deep water.

\section{Solar water purification based on the AFPCF}

To explore the water purification capacity of the AFPCF, two samples including simulated seawater containing five primary ions $\left(\mathrm{Na}^{+}, \mathrm{Mg}^{2+}, \mathrm{K}^{+}, \mathrm{Ca}^{2+}\right.$ and $\left.\mathrm{Pb}^{2+}\right)$ and waste water containing four types of bacteria (E. coli, S. epidermidis, S. aureus, MRSA) were carefully prepared. In order to collect purified water from the samples, we also hand-made a closed evaporation system, which consists of a glass cover with high transmittance, a Dewar Flask filled with sample water and the evaporator (Fig. 4A). We first used the simulated seawater to conduct the test, as the evaporation system under the tilted irradiation of 1 sun, vapor generated and condensed on the inner wall of the glass cover (Fig. S12). With the increase of the irradiation time, the vapor became liquid and formed droplets which merged together (Fig. 4B). After purification by the solar driven evaporation system, the light yellow-colored simulated seawater was transferred into a colorless and clear liquid (Fig. S13). As noted by inductively coupled plasma mass spectrometry (ICP-MS), the concentration of ions $\left(\mathrm{Na}^{+}, \mathrm{Mg}^{2+}, \mathrm{K}^{+}, \mathrm{Ca}^{2+}\right.$ and $\left.\mathrm{Pb}^{2+}\right)$ in the simulated seawater decreased from $10^{3}$ to $10^{-1} \mathrm{mg} \mathrm{L}^{-1}$ with an efficiency of $99.9 \%$ after solar evaporation treatment, which was much lower than the World Health Organization (WHO) standard for drinking water (1\%o) (Fig. 4C). (31) Apart from ions pollutants, bacteria and microorganism pollution is another challenge for water purification. We then utilized the evaporation system to purify the waste water containing four types of bacteria. As shown in Fig. 4D and 4E, there is no any bacterial clone could be observed in the condensed water, showing the efficient removal of the bacteria. These results strongly suggested that this developed AFPCF evaporator was capable of achieving water purification from seawater and waste water via solar irradiation.

\section{Conclusion}

In summary, an advanced 3D evaporator, namely AFPCF, that combines side area-assisted evaporation with a "One Stone Two Birds" AIEgen is developed to realize excellent evaporation property and antibiofouling performance. AFPCF evaporator exhibits interconnected pore and large side area, which are beneficial for vapor escaping and gaining energy from tilted sunlight irradiation. The presence of the tactfully designed AIEgen endows the evaporator with excellent photothermal conversion and photodynamic antibacterial capacity. Notably, solar evaporation rate of the evaporator is determined to be as high as $3.6 \mathrm{~kg} \mathrm{~m}^{-2} \mathrm{~h}^{-1}$, which is far beyond the theoretical limit (1.62 $\left.\mathrm{kg} \mathrm{m}^{-2} \mathrm{~h}^{-1}\right)$ under 1 sun of irradiation. Moverover, the continuous ROS generation of the AIEgen under the irradiation of sunlight highly inhibits the growth of bacteria nearby the 
evaporator, revealing the superior antibiofouling performance. The presented evaporator is further used to purify simulated seawater and waste water containing various bacteria, and achieved a high quality purification meeting the WHO standards for drinking water. Therefore, the multifunctional side area-assisted evaporator opened a window to construct next generation of smart evaporator for water purification and other applications, including moisture management, Marine ecosystem, and sewage treatment.

\section{Materials and Methods}

Chemicals and materials: Poly(methyl methacrylate) (PMMA) (Mw = 120 000), tetrahydrofuran (THF), N, N-dimethylformamide (DMF), and sodium borohydride were all obtained from Merck (Darmstadt, Germany). 4,7-dibromobenzo[c][1,2,5]thiadiazole, benzil, $\mathrm{Pd}(\mathrm{PPh} 3) 4$, 4-methoxy-N(4-methoxyphenyl) -N-(4-(4,4,5,5-tetramethyl-1,3,2-dioxaborolan-2-yl)phenyl)aniline were purchsed from Bidepharm (Shanghai, China). All the chemicals used as received without further purification. Luria-Bertani (LB) broth and LB agar were from USB Co. Zinc dust. Phosphate buffer saline (PBS) was from Sigma-Aldrich. E. coli (ATCC 25922), S. epidermidis (ATCC) and S. aureus (ATCC) were from ATCC. MRSA were from Beijing Tiantan Hospital (China), Milli-Q water was from a Milli-Q purification system (Merck Millipore, Germany).

Preparation of TPA-BTDH/PMMA nanofibrous mat: For electrospun TPA-BTDH/PMMA nanofibers, the solution for electrospinning was prepared by dissolving $1.0 \mathrm{~g}$ of PMMA in $10 \mathrm{~mL}$ mixture of THF and DMF (v/v = 7:3) at a final concentration of $10 \mathrm{wt} \%$, and then $50 \mathrm{mg}$ of TPABTDH powders were added into the as-prepared solution. The solution was loaded into a 5-mL plastic syringe with a 21-gauge needle attached and dispensed using a KD Scientific syringe pump. The injection rate was set at $1 \mathrm{~mL} / \mathrm{h}$. A voltage of $13 \mathrm{kV}$ generated by Gamma High Voltage was applied to the needle, while the distance between the tip of the needle to the collector was set to $15 \mathrm{~cm}$, electrospun time was set to $12 \mathrm{~h}$.

Fabrication of AFPCF: TPA-BTDH/PMMA nanofibrous mats were cut into a circle with the diameter of $3 \mathrm{~cm}$, and then treated by plasma for $5 \mathrm{~min}$ to improve their hydrophilicity. During expansion, the prepared nanofibrous mats were immersed in $100 \mathrm{~mL} \mathrm{NaBH4}$ solution (1 M) for varying of time $(0,10,30$ and $60 \mathrm{~min})$ at room temperature. Following expansion, the $\mathrm{NaBH} 4$ solution was discarded and the expanded TPA-BTDH/PMMA nanofiber foam were gently transferred into a beaker and rinsed three times with deionized water to remove sodium salt.

Characterization: The SEM images of samples were observed using a FEI APREOS field emission scanning electron microscopy (FE-SEM) (Thermal, Japan). The IR thermal images and digital photographs were taken by E6 IR-camera (FLIR, USA) and mate-20 pro mobile phone 
(HUAWEI, China), respectively. 1H spectra were measured on Bruker AVANCE III 600M NMR spectrometers. Absorbance spectra were recorded using PerkinElmer Lambda 950 (PERKINELMER, USA). The concentration of ions was tracked by ICP-OES (ICAP 7000, USA) with dilutions in $2 \% \mathrm{HNO}_{3}$ to make the loaded ion concentration lower than 10 parts per million.

Solar steam generation measurements: The experiment of photothermal properties was performed by a Solar-500L solar simulator system (NBET, Beijing), which contains a solar simulator and an AM 1.5G light filter. The solar density is calculated by a VLP-2000 light power meter (LASER, China). The temperature changes of the samples were taken by E6 IR-camera (FLIR, USA). For tilted irradiation test, the irradiation direction was set as tilted to the evaporator with incident angle of $60^{\circ}$, to ensure that the surface and side area of AFPCF can be fully irradiated. The mass change of water was was measured via a PR224ZH high accuracy balance (OHAUS, New Jersey).

Antibacterial Activity Assay: AFPCF samples were cut into $1.0 \mathrm{~cm}$ in diameter and placed in a 48-well plate. The samples were challenged with E. coli, S. epidermidis, S. aureus and MRSA bacteria at a concentration of $1 \times 106 \mathrm{CFU} \mathrm{mL}-1.1 \times 107 \mathrm{CFU} \mathrm{mL}-1$ bacteria were dispersed in 1 $\mathrm{mL}$ PBS solution. $100 \mu \mathrm{L}$ of the bacteria solution was added into 48 -well plate. E. coli, S. epidermidis, S. aureus and MRSA after being treated with or without AFPCF-3cm under dark condition and simulated sunlight irradiation for $10 \mathrm{~min}$ after static $10 \mathrm{~min}$. Last, $50 \mu \mathrm{L}$ bacteria solution spread onto a LB agar plate and incubated at $37{ }^{\circ} \mathrm{C}$ for $24 \mathrm{~h}$. Colonies were counted and colony forming unit (CFU mL-1) were calculated. Experiments were performed with three replicates.

AFPCF samples were cut into $3.0 \mathrm{~cm}$ in diameter and placed in a 6-well plate. The samples were challenged with E. coli, S. aureus and MRSA bacteria at a concentration of $1 \times 108 \mathrm{CFU}$ $\mathrm{mL}-1.100 \mu \mathrm{L}$ of the bacteria solution was added into 6-well plate and then $4 \mathrm{~mL}$ PBS was added to prevent water loss from the sunlight. $100 \mu \mathrm{L}$ bacteria solution was spread onto a LB agar plate and incubated at $37{ }^{\circ} \mathrm{C}$ for $24 \mathrm{~h}$ after nature sun irradiation for $60 \mathrm{~min}$. Colonies were counted and $\mathrm{CFU} \mathrm{mL}-1$ were calculated. Experiments were performed with three replicates.

\section{References}

1. P. Tao, G. Ni, C. Song, W. Shang, J. Wu, J. Zhu, G. Chen, T. Deng, Solar-driven interfacial evaporation. Nat. Energy 3, 1031-3041 (2018). 
2. F. Zhao, Y. Guo, X. Zhou, W. Shi, G. Yu, Materials for solar-powered water evaporation. Nat.

13. X. Zhou, F. Zhao, Y. Guo, B. Rosenberger, G. Yu, Architecting highly hydratable polymer networks to tune the water state for solar water purification. Sci. Adv. 5, eaaw5484 (2019).

14. N. Xu, J. Li, Y. Wang, C. Fang, X. Li, Y. Wang, L. Zhou, B. Zhu, Z. Wu, S. Zhu, J. Zhu, A water lily-inspired hierarchical design for stable and efficient solar evaporation of highsalinity brine. Sci. Adv. 5, eaaw7013 (2019)

15. L. Zhou, Y. Tan, J. Wang, W. Xu, Y. Yuan, W. Cai, S. Zhu, J. Zhu, 3D self-assembly of aluminium nanoparticles for plasmon-enhanced solar desalination. Nat. Photon. 10, 393-398 (2016). 
16. Y. Kuang, C. Chen, S. He, E. M. Hitz, Y. Wang, W. Gan, R. Mi, L. Hu, A high-performance self-regenerating solar evaporator for continuous water desalination. Adv. Mater. 31, 1900498 (2019).

17. H. Li, H. Wen, Z. Zhang, N. Song, R. T.K. Kowk, J. W. Y. Lam, L. Wang, D. Wang, B. Z. Tang, Reverse thinking of the aggregation-induced emission principle: amplifying molecular motions to boost photothermal efficiency of nanofibers. Angew. Chem. Int. Ed. 59, 2037120375 (2020).

18. H. Li, H. Wen, J. Li, J. Huang, D. Wang, B. Z. Tang, Doping AIE photothermal molecule into all-fiber aerogel with self- pumping water function for efficiency solar steam generation. ACS Appl. Mater. Interfaces 12, 26033-26040 (2020).

19. C. Liu, K. Hong, X. Sun, A. Natan, P. Luan, Y. Yang, H. Zhu, An 'antifouling' porous loofah sponge with internal microchannels as solar absorbers and water pumpers for thermal desalination. J. Mater. Chem. A 8, 12323-12333 (2020).

20. Y. Xu, J. Ma, Y. Han, H. Xu, Y. Wang, D. Qi, W. Wang, A simple and universal strategy to deposit Ag/polypyrrole on various substrates for enhanced interfacial solar evaporation and antibacterial activity. Chem. Eng. J. 384, 123379 (2020).

21. X. Y. Wang, J. Xue, C. Ma, T. He, H. Qian, B. Wang, J. Liu, Y. Lu, Anti-biofouling doublelayered unidirectional scaffold for long-term solar-driven water evaporation. J. Mater. Chem. A 7, 16696-16703 (2019).

22. C. Chen, Y. Kuang, L. Hu, Challenges and opportunities for solar evaporation. Joule 3, 683718 (2020).

23. V. D. Dao, N. H. Vu, S. Yun, Recent advances and challenges for solar-driven water evaporation system toward applications. Nano Energy 68, 104324 (2020).

24. X. J. Zha, X. Zhao, J. H. Pu, L. S. Tang, K. Ke, R. Y. Bao, L. Bai, Z. Y. Liu, M. B. Yang, W. Yang, Flexible anti-biofouling MXene/cellulose fibrous membrane for sustainable solardriven water purification. ACS Appl. Mater. Interfaces 11, 36589-36597 (2019).

25. M. Kang, Z. Zhang, N. Song, M. Li, P. Sun, X. Chen, D. Wang, B. Z. Tang, Aggregation enhanced theranostics: AIE sparkles in biomedical field. Aggregate 1, 80-106 (2020).

26. W. Xu, D. Wang, B. Z. Tang, Angew. Chem. Int. Ed. 2020, DOI: 10.1002/anie.202005899.

27. Z. Zhang, W. Xu, M. Kang, H. Wen, H. Guo, P. Zhang, L. Xi, L. Wang, D. Wang, B. Z. Tang, An all-round athlete on the track of phototheranostics: subtly regulating the balance between radiative and nonradiative decays for multimodal imaging-guided synergistic therapy. Adv. Mater. 32, 2003210 (2020). 
28. W. Xu, M. M. S. Lee, J. J. Nie, Z. Zhang, R. T. K. Kwok, J. W. Y. Lam, F. J. Xu, D. Wang, B.

Z. Tang, Three-pronged attack by homologous far-red/NIR AIEgens to achieve $1+1+1>3$ synergistic enhanced photodynamic therapy. Angew. Chem. Int. Ed. 59, 9610-9616 (2020).

29. S. Chen, J. V. John, A. McCarthy, M. A. Carlson, X. Li, J. Xie, Fast transformation of 2D nanofiber membranes into pre-molded 3D scaffolds with biomimetic and oriented porous structure for biomedical applications. Appl. Phys. Rev. 7, 021406 (2020).

30. G. Ni, G. Li, S. V. Boriskina, H. Li, W. Yang, T. Zhang, G. Chen, Steam generation under one

sun enabled by a floating structure with thermal concentration. Nat. Energy 1, 16126 (2016).

31. F. Edition, Guidelines for drinking-water quality. WHO Chron. 38, 104-108 (2011).

Acknowledgments: We thank Instrumental Analysis Center of Shenzhen University for SEM analysis. Funding: This work was partially supported by the Natural Science Foundation for Distinguished Young Scholars of Guangdong Province (2020B1515020011), the Natural Science Foundation of China $(21801169,22005195)$, the Science and Technology Foundation of Shenzhen City (JCYJ20190808153415062), and China Postdoctoral Science Foundation (2020M672797). Author contributions: H. L., D. W., and B. Z. T. conceived and designed the experiments. W. Z. performed the synthesis AIE agent. H. L. conducted the fabrication of evaporator, solar steam generation test and collected the data. M. L., and Y. L. performed antibacterial experiment. H. L., W. Z., R. T. K. Kwok., J. W. Y. Lam., L. W., and D. W performed data analysis, H. L., D. W., and B. Z. T. co-wrote and revised the paper. All authors discussed the results and approved the final version of the manuscript. Competing interests: The authors declare that they have no competing interests. Data and materials availability: All data needed to evaluate the conclusions in the paper are present in the paper and/or the Supplementary Materials. Additional data related to this paper may be requested from the authors. 

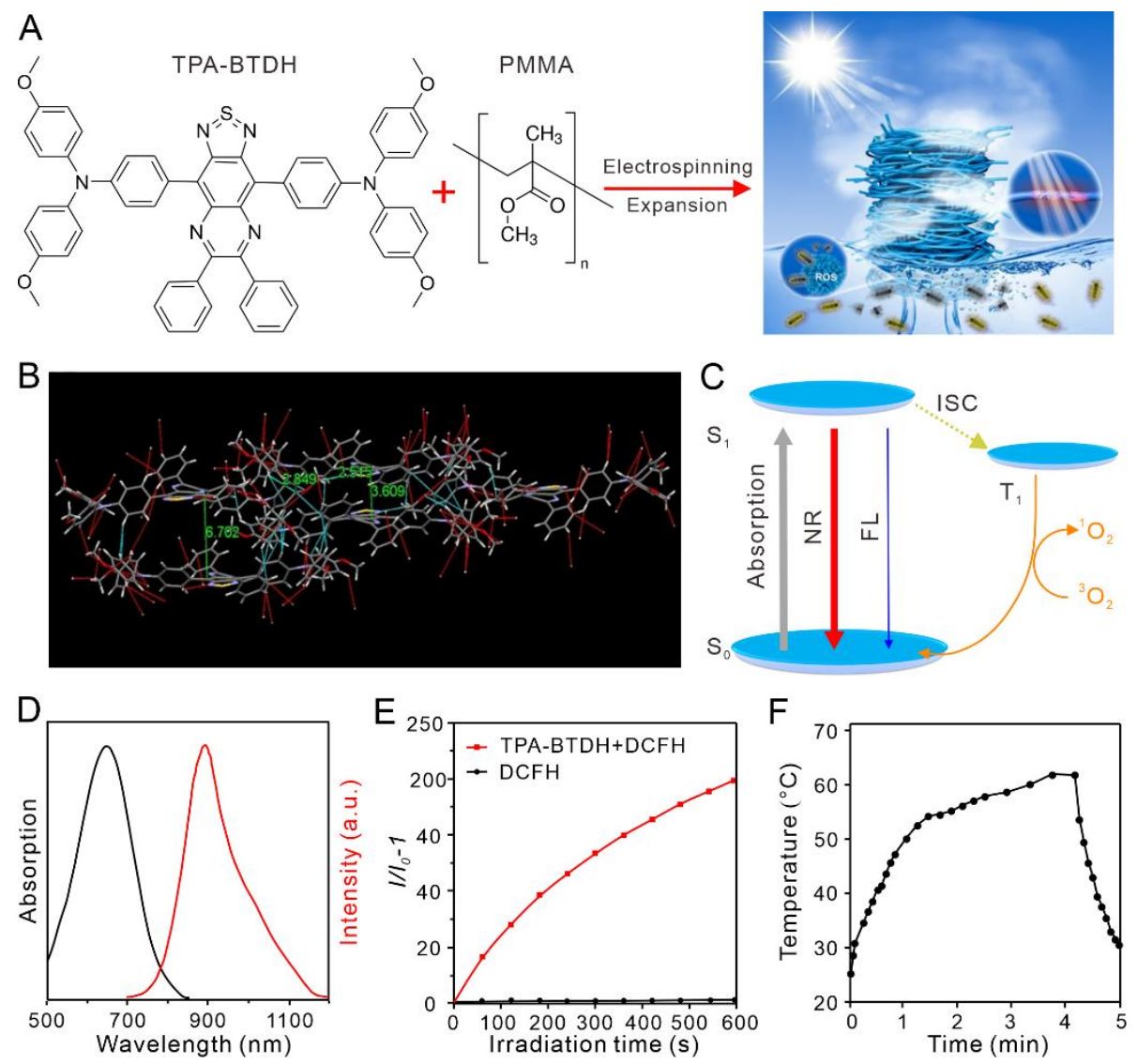

Fig. 1. Synthesis and characterization of AIEgens. (A) The structure of TPA-BTDH and a schematic shows the design concept of the side area-assisted evaporator. (B) The intermolecular plane distances and various intermolecular and intramolecular interactions of TPA-BTDH via Single-crystal X-ray analysis. (C) Jablonski diagram illustrating excited-state energy dissipations of TPA-BTDH. (D) Absorption and PL spectra of TPA-BTDH in THF solution. (E) ROS generation of TPA-BTDH upon xenon lamp irradiation using dichlorofluorescin (DCFH). (F) The temperature changes of TPA-BTDH powder as it was exposed to 1 sun of irradiation. 
A

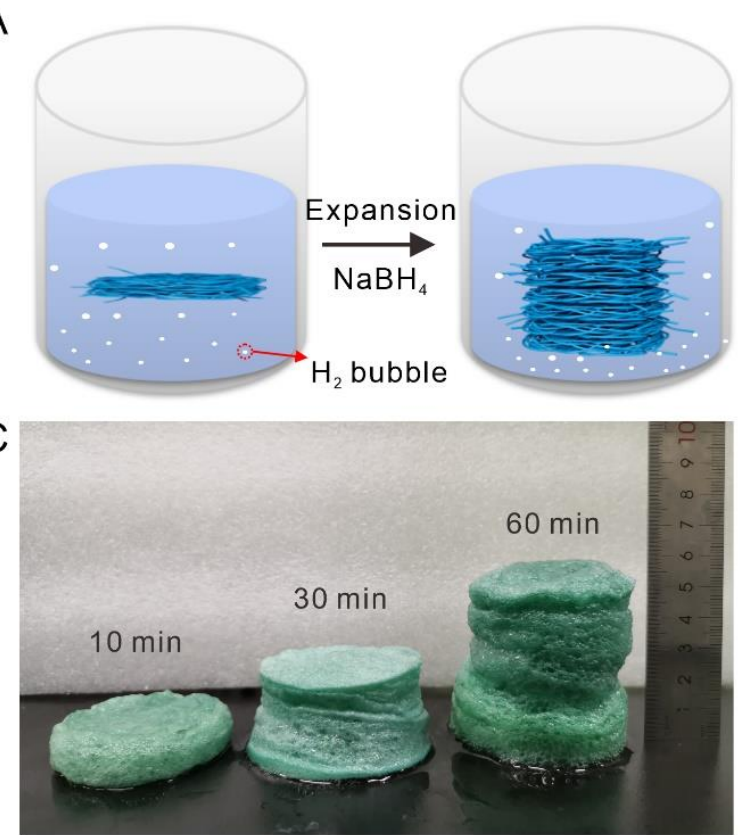

E

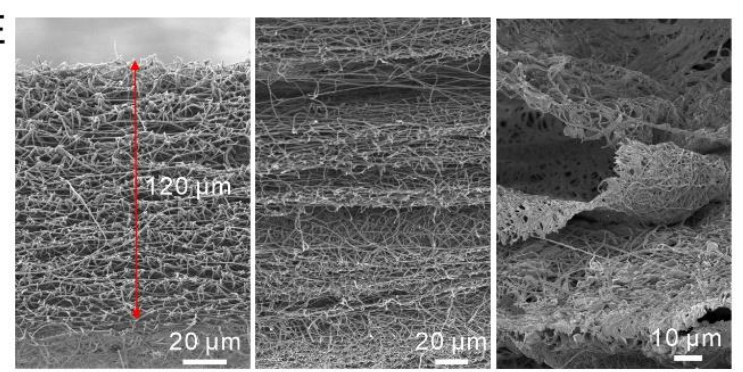

B
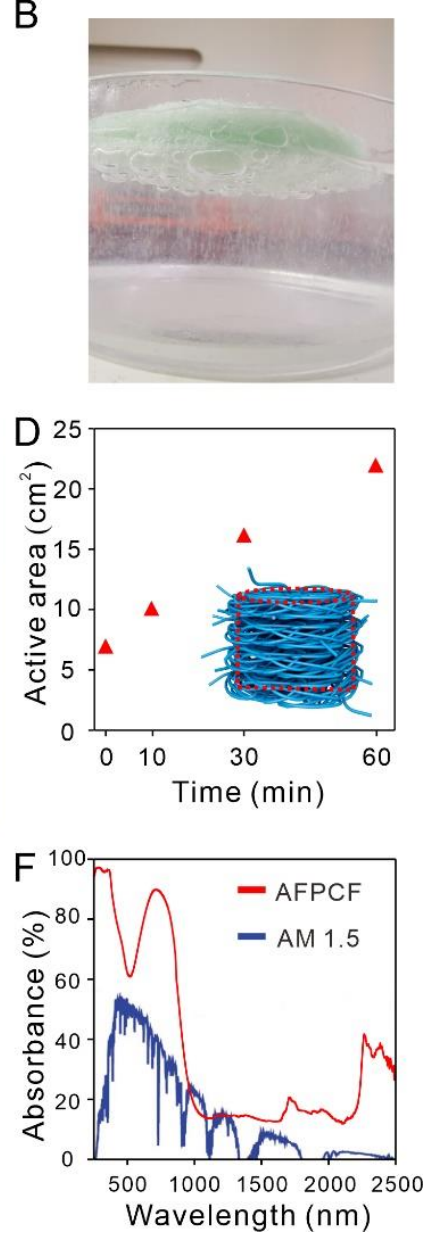

Fig. 2. Fabrications and characterizations of the 3D AFPCF. (A) Schematic illustration of the expansion process from 2D nanofibrous mat to $3 \mathrm{D}$ structure. (B) A photograph of generated bubbles as a TPA-BTDH/PMMA nanofibrous mat was placed into $1 \mathrm{M} \mathrm{NaBH}_{4}$ solution. (C) Photo images of the TPA-BTDH/PMMA nanofibrous mat after the treatment with $1 \mathrm{M} \mathrm{NaBH}_{4}$ solution for 10,30 and 60 min, respectively. (D) Active area of AFPCF was calculated (surface area plus one side area) after the treatment with $1 \mathrm{M} \mathrm{NaBH}_{4}$ solution for 10, 30 and $60 \mathrm{~min}$, respectively. (E) SEM images showing cross section morphologies of TPABTDH/PMMA nanofibrous mat before and after the treatment of $1 \mathrm{M} \mathrm{NaBH}_{4}$ solution for 10 and $60 \mathrm{~min}$. (F) The absorption spectra of the AFPCF ranging from 250 to $2500 \mathrm{~nm}$ and solar spectral irradiance weighted by standard AM 1.5G solar spectrum. 

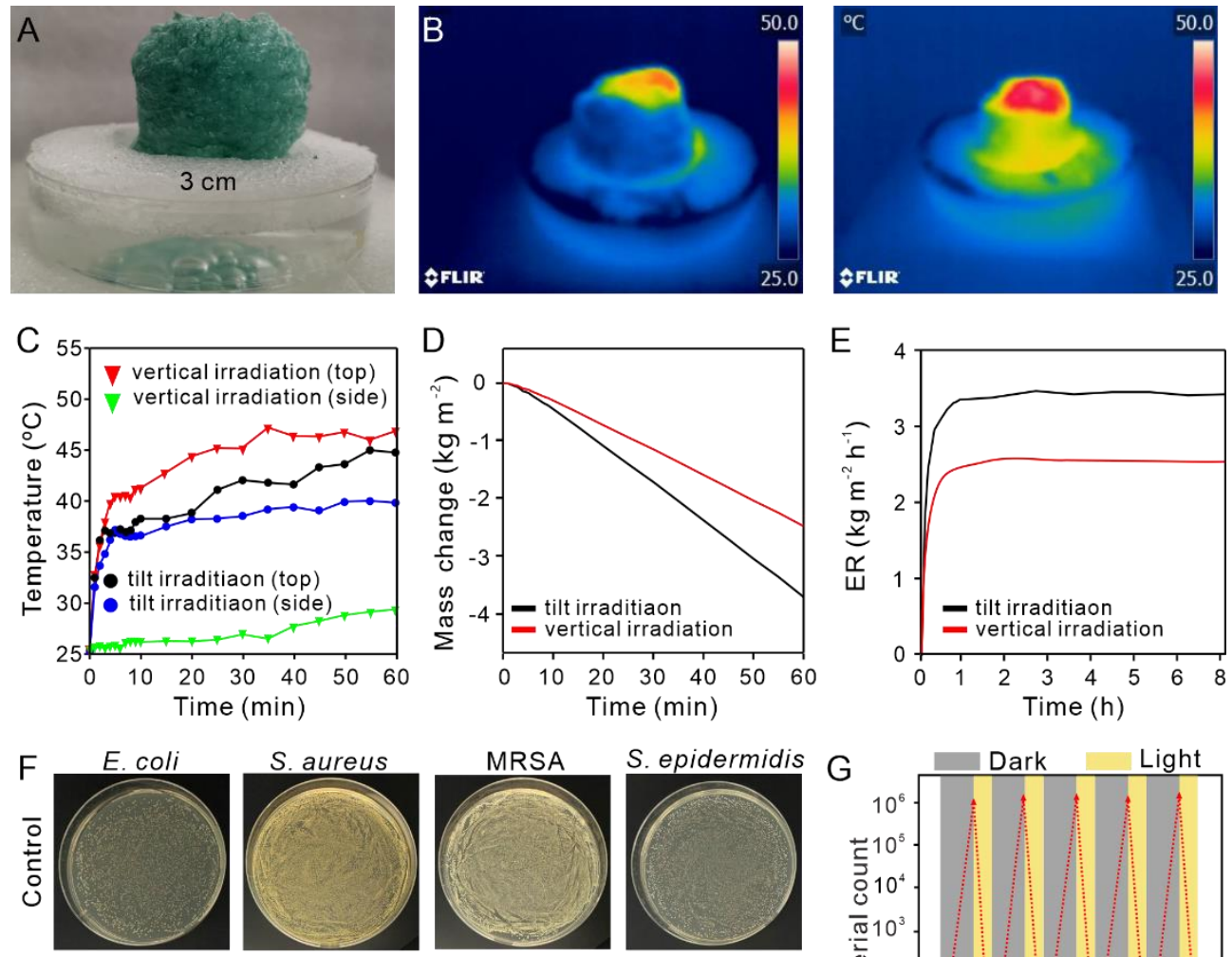

S. epidermidis
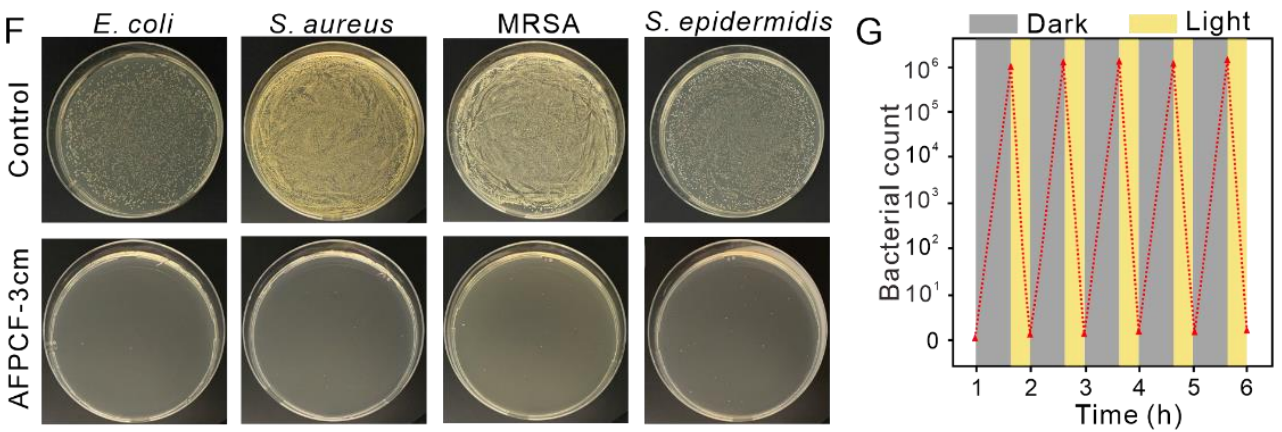

Fig. 3. Performance of solar-steam generation and anti-bacterial. (A) A photo image of AFPCF with height of $3 \mathrm{~cm}$ enclosed by foam and floating on the water. (B) IR thermal images showing temperature changes of the AFPCF with height of $3 \mathrm{~cm}$ under vertical and tilted irradiation of 1 sun. (C) A comparison between the temperature rise recorded from the surface and side of AFPCF upon the vertical and tilted irradiation of the 1 sun for $60 \mathrm{~min}$. (D) The mass loss of water and (E) evaporation rate of AFPCF with height of $3 \mathrm{~cm}$ under vertical and tilted irradiation of 1 sun for period time. (F) Photographs of E. coli, S. aureus, MRSA and $S$. epidermidis cultured on agar plate supplemented with TCP and AFPCF-3 cm under simulated sunlight for 10 min. (G) Five cycle antibacterial test of AFPCF under repeated simulated sunlight irradiation and dark condition. Each cycle, $1 \times 10^{6} \mathrm{CFU}$ $\mathrm{mL}^{-1}$ E. coli was pipetted on the AFPCF before the dark condition. 

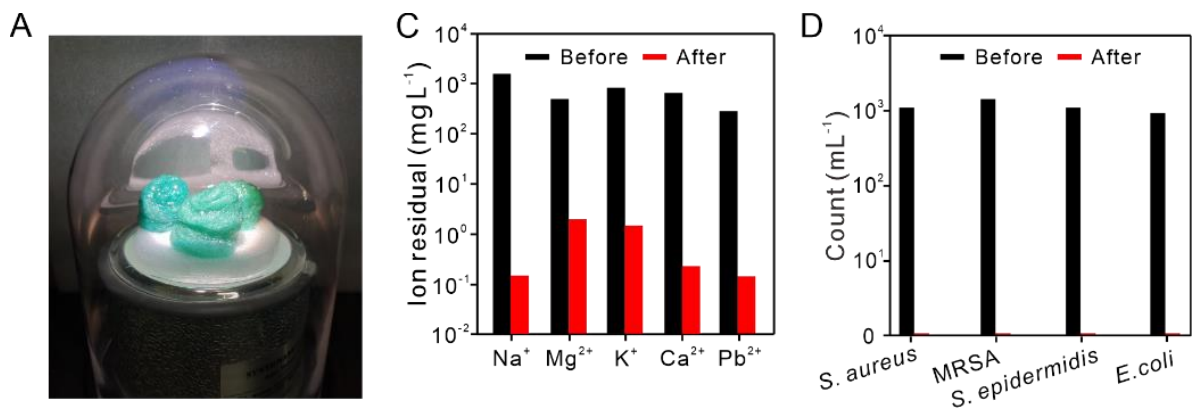

$\mathrm{B}$

E
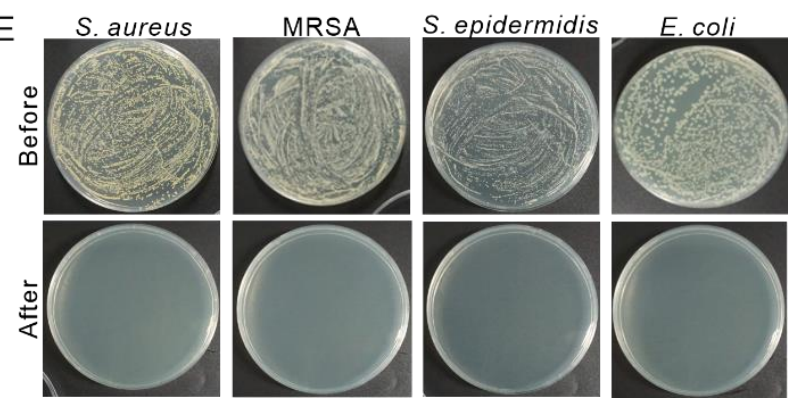

Fig. 4. Solar water purification based on the AFPCF. (A) A photograph of the handmand solar steam generation device containing a glass cover, a AFPCF evaporator, and a Dewar Flask filled with seawater or waste water. (B) A photograph showing clean water condensed on the inner wall of the glass cover. (C) The concentrations of ions $\left(\mathrm{Na}^{+}, \mathrm{Mg}^{2+}, \mathrm{K}^{+}, \mathrm{Ca}^{2+}\right.$ and $\left.\mathrm{Pb}^{2+}\right)$ in the simulated seawater and the collected clean water after evaporation. (D) The counts of bacterial clones in the simulated wastewater and the collected water after evaporation. (E) Photographs of S. aureus, MRSA, S. epidermidis and E. coli cultured on agar plate which are collected from the evaporated water. 


\section{Supplementary Materials}

Supplementary material for this article is available at

Supplementary Text

Figs. S1. Synthetic route to compound TPA-BTDH

Figs. S2. Single-crystal X-ray and calculated analysis of TPA-BTDH

Figs. S3. Fabrication and characterization of TPA-BTDH/PMMA nanofibrous mat.

Figs. S4. SEM images of TPA-BTDH/PMMA nanofibers.

Figs. S5. Temperature changes of the AFPCF in dry state under the irradiation of 1 sun.

Figs. S6. Solar steam generation performances of AFPCF.

Figs. S7. Temperature changes the surface of AFPCF with height of $1 \mathrm{~cm}$ under vertical and tilted irradiation of 1 sun.

Figs. S8. Anti-bacterial capacities of AFPCF under the simulated sunlight.

Figs. S9. Formed viable colony units of E. coli, S. epidermidis, S. aureus and MRSA after being treated with or without AFPCF under dark condition for $10 \mathrm{~min}$, and then spread onto agar plate and incubated at $37^{\circ} \mathrm{C}$ for $24 \mathrm{~h}$.

Figs. S10. SEM images of $E$. coli and $S$. aureus after being treated AFPCF under simulated sunlight and dark condition for $10 \mathrm{~min}$.

Figs. S11. Anti-bacterial capacities of AFPCF under the sunlight.

Figs. S12. Photograph showing the detailed process of the vapor condensed on the inner wall of the glass cover.

Figs. S13. Photograph of the collected vapor.

Tables S1. Crystal data and structure refinement for TPA-BTDH. 